Musées, Patrimoine et Culture scientifiques et techniques

$158 \mid 2015$

mars-avril 2015

\title{
À la recherche de nouveaux publics : quand la chimie s'invite au musée
}

Arnaud Quertinmont et Marie-Aude Laoureux

\section{OpenEdition}

\section{Journals}

Édition électronique

URL : http://journals.openedition.org/ocim/1499

DOI : 10.4000/ocim.1499

ISSN : 2108-646X

Éditeur

OCIM

Édition imprimée

Date de publication : 1 mars 2015

Pagination : 25-30

ISSN : 0994-1908

Référence électronique

Arnaud Quertinmont et Marie-Aude Laoureux, «À la recherche de nouveaux publics : quand la chimie s'invite au musée », La Lettre de I'OCIM [En ligne], 158 | 2015, mis en ligne le 01 mars 2016, consulté le 14 novembre 2019. URL : http://journals.openedition.org/ocim/1499 ; DOI : 10.4000/ocim.1499

Ce document a été généré automatiquement le 14 novembre 2019.

Tous droits réservés 


\title{
À la recherche de nouveaux publics : quand la chimie s'invite au musée
}

\author{
Arnaud Quertinmont et Marie-Aude Laoureux
}

Atelier olfactif réalisé à l'occasion de l'exposition Parfums d'Antiquité. La rose et l'encens.

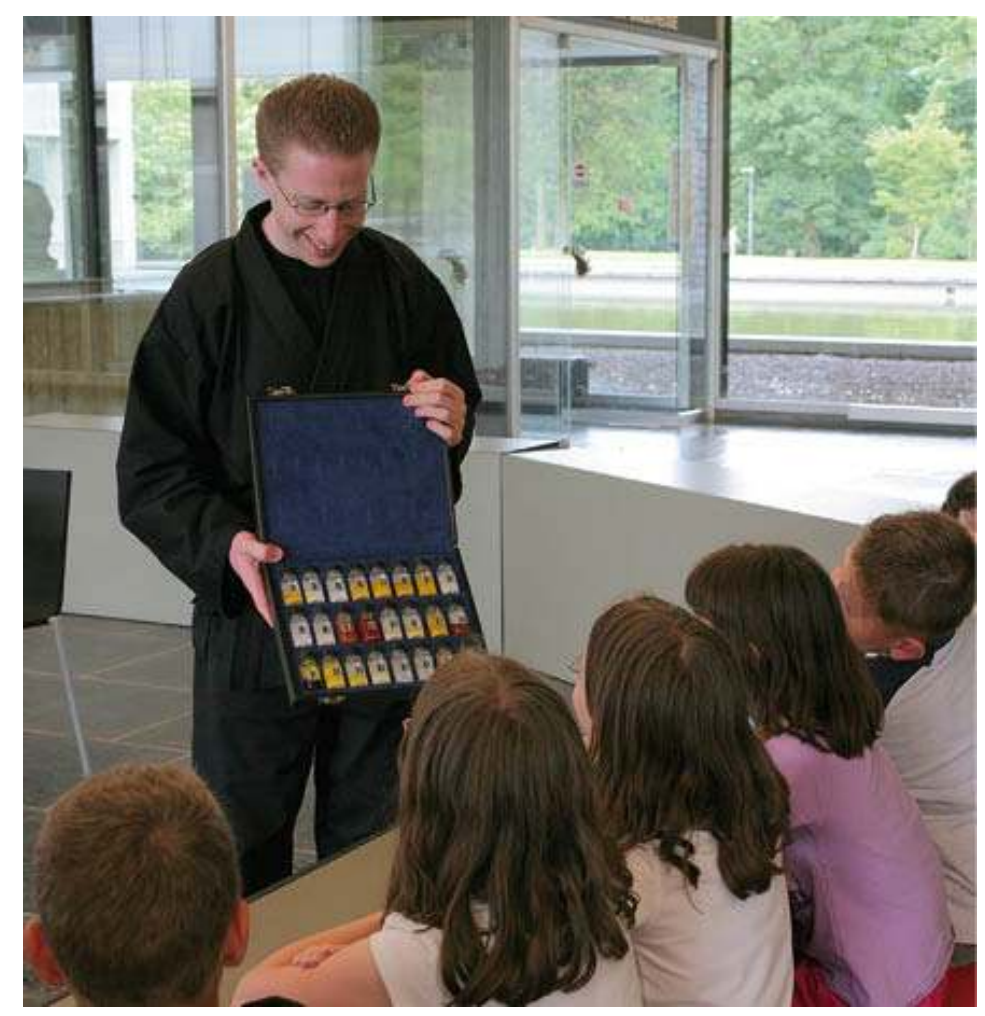

(c) Musée royal de Mariemont

En Belgique francophone, au sein de l'asbl MSW, principale fédération des musées en Wallonie, les musées sont répartis en quatre grands réseaux : Art\&Mus (musées d'art), ArchéoPass (musées d'archéologie), HOmusée (musées d'ethnologie) et PISTe 
(Patrimoine industriel, scientifique et technique). Cette structure en réseau permet de travailler avec d'autres institutions aux thématiques assez proches et d'établir des synergies entre les différentes activités réalisées. Au premier abord, on pourrait croire que cette structure est assez rigide et compartimente les médiations et les publics. La réalité est heureusement toute autre.

2 L'une des spécificités du musée royal de Mariemont est d'être à la fois membre du réseau ArchéoPass et du réseau Art\&Mus. Cet engagement reflète la diversité de nos collections, à la fois artistiques, historiques, archéologiques et bibliophiles. Lorsque nous proposons une activité, nous ne nous interrogeons pas sur sa nature, plus archéologique ou plus artistique. Traditionnellement, le public scolaire qui nous est fidèle est composé de classes de latin, de grec et d'histoire. Ces dernières années, grâce aux thématiques variées des expositions du musée, nous avons eu l'opportunité d'interroger nos méthodes de médiation et d'investiguer de nouvelles façons d'approcher les collections d'un musée tel que le nôtre ${ }^{1}$. Cela a également été l'occasion de tenter de décloisonner les réseaux de façon à les ouvrir à d'autres disciplines scolaires.

3 Il en a résulté la création d'outils (visites, ateliers, supports didactiques) permettant d'aller ainsi à la rencontre de nouveaux publics. Nous souhaitons que le musée devienne, comme l'écrit M.-É. Ricker, « l'objet d'exploitations bien plus diversifiées que celles strictement liées aux matières artistiques et historiques $»^{2}$. Pour atteindre cet objectif, nous devons reconnaître la limite de nos compétences et faire appel à des spécialistes.

\section{Des ateliers multidisciplinaires}

4 L'exposition Parfums de l'Antiquité. La rose et l'encens, en 2006 a ainsi été l'occasion de nous pencher sur le métier de parfumeur, d'esthéticienne et de professionnels de la beauté. Une médiation adaptée a permis de mettre en avant les multiples implications psychologiques et anthropologiques que revêtent les parfums, trop souvent limités à leur côté éphémère, volatile et superficiel. C'est grâce à un partenariat avec un parfumeur professionnel que nous pouvons proposer, depuis 2006, des «ateliers olfactifs » durant lesquels les visiteurs restituent les odeurs de l'Antiquité dans une dimension historique, sociologique et culturelle. Suite à cette exposition, nous avons développé un parcours dans les collections permanentes, ce qui nous a permis de fidéliser, entre autres, plusieurs groupes d'étudiants esthéticiens. Cette approche sensorielle permet de s'écarter de la vue et de faire appel à l'odorat, au goût ou encore au toucher. Cette visite est dès lors également adaptée aux groupes de visiteurs porteurs d'un handicap.

5 Avec l'exposition Le Verre Art Déco en 2011, nous sommes partis à la rencontre de souffleurs de verre et d'artisans verriers. Un atelier « Laisser toucher » a alors été créé. Cet atelier nous permet de prolonger la visite de l'exposition en abordant les matières premières, les outils, les techniques, les gestes de l'artisan... autant de thématiques que l'on s'attendrait plutôt à trouver dans un musée des sciences et techniques.

6 En 2012, dans le cadre de l'exposition Écrivains: modes d'emploi. De Voltaire à bleuOrange, nous nous sommes entourés d'enseignants de langue et littérature française afin de proposer un parcours permettant aux étudiants d'appréhender le 
travail de l'écrivain, d'évoquer le rapport à l'écrit dans une société submergée par les nouvelles technologies et les nouveaux moyens de communication. La question du support d'écriture a également été abordée par une exposition intitulée Illuminations. Aventure du livre en Occident. Comprendre le rôle du livre dans notre histoire, c'est par conséquent découvrir les enjeux économiques, politiques et culturels qui lui ont permis d'exister, et c'est aussi comprendre les évolutions qui témoignent de nouvelles préoccupations et de nouveaux besoins. Aujourd'hui encore, l'ensemble de ces choix influence notre manière de concevoir et d'utiliser le livre.

7 L'exposition Trésor? / Trésor ! Archéologie au cœur de l'Europe qui s'est tenue fin 2014 nous a donné une autre occasion d'inviter les sciences au musée. Nous pouvons ainsi proposer aux élèves du secondaire des ateliers de réflexion, d'observation et d'expérimentation visant à expliquer les sciences partenaires de l'archéologie comme l'archéozoologie, la xylologie...

\section{L'Égypte et les sciences : de nouveaux dispositifs de transmission}

8 Depuis près de 15 ans, «Destination Sciences ", la cellule scientifique de la Maison de la Laïcité de La Louvière, organise des évènements qui ont pour objectif de désamorcer la désaffection des jeunes envers les carrières scientifiques en brisant le cliché du chercheur poussiéreux et en mettant en évidence le fait que les sciences font partie de notre quotidien et que les connaissances scientifiques font partie intégrante de la culture générale.

9 Par le biais d'un concours annuel (celui de 2013 a rassemblé plus de 140 participants), les organisateurs de « Destination Sciences » visent à :

10 - amener les étudiants du secondaire à devenir acteurs de leur savoir plutôt que spectateurs : ce sont eux qui recherchent les informations, les trient, les choisissent... et les présentent ;

11 - appliquer une démarche scientifique, notamment en leur faisant prendre conscience qu'il vaut mieux présenter une expérience ratée, en sachant expliquer pourquoi plutôt qu'une expérience qui réussit sans rien y comprendre ;

12 - découvrir des conditions de travail proches de celles de la vie professionnelle (apprendre à prévoir et respecter des délais, travailler en équipe, réaliser une médiation scientifique envers le grand public).

13 C'est dans le cadre du concours 2014, intitulé « Sciences et Beauté » que nous avons été contactés par un professeur de chimie qui désirait venir au musée avec un groupe d'étudiants de l'enseignement secondaire supérieur. Ceux-ci avaient décidé de s'intéresser au khôl égyptien et souhaitaient en savoir plus. En effet, des études récentes ont permis de démontrer la connaissance empirique qu'avaient les Égyptiens de certaines démarches de « synthétisations chimiques ». Nous avons alors proposé une médiation culturelle ciblée sur ce produit du point de vue des textes, de l'iconographie ainsi que du matériel et des données connus par l'archéologie. 


\section{Le khôl et les sciences historiques}

L'oeil égyptien fardé, souligné et rehaussé d'un trait de khôl occupe une place particulière dans l'imaginaire collectif moderne. Symbolisant parfois à lui seul la civilisation de l'Égypte ancienne, l'oudjat, l'œil d'Horus fait appel à de nombreuses références mythologiques.

Affiche du concours "Sciences et beauté ».

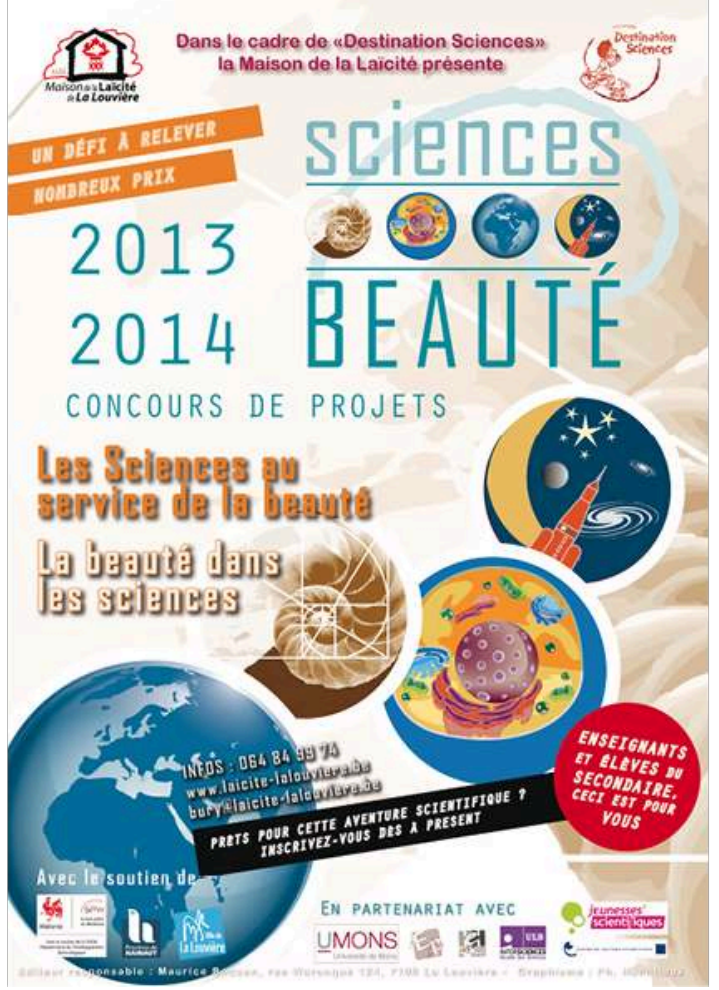

(c) Maison de la Laïcité de La Louvière contre son oncle Seth pour conquérir le trône qui lui revenait de droit. Au cours du combat, Seth lui arracha l'œil gauche, le découpa en morceaux et jeta ceux-ci dans le Nil. À l'aide d'un filet, Thot repêcha tous les morceaux sauf un. Il remplaça magiquement le fragment manquant permettant ainsi à l'œil de fonctionner de nouveau. L'amulette oudjat, l'un des symboles de protection les plus forts de l'Égypte antique, garantit ainsi à son porteur qu'il conservera son intégrité physique dans le monde des vivants et dans l'au-delà.

Grâce à certains textes, nous connaissons le procédé de fabrication du khôl égyptien, qu'il s'agisse du fard vert ouadj composé de chrysocolle (silicate de cuivre) ou de malachite réduit en poudre et mélangé à un corps gras; ou encore du fard noir mesdemet composé de sels de plomb. Le Papyrus Ebers, datant du Nouvel Empire (16e siècle av. J.-C.) et aujourd'hui conservé à la bibliothèque universitaire de Leipzig, nous apprend également que pour les anciens Égyptiens le khôl revêtait une importance magique et médicale: "Remède pour soigner la vue: galène, ocre rouge, [nom de plante], partie mâle de la galène. Sera broyé finement et préparé en une masse homogène et appliquée sur les yeux ». Dire l'incantation "Viens malachite! Viens 
malachite! Viens la verte! Viens écoulement de l'œil d'Horus ! Viens rejet de l'œil d'Atoum! Viens sécrétion sortie d'Osiris! »".

Masque funéraire égyptien (détail) montrant l'utilisation du khôl.

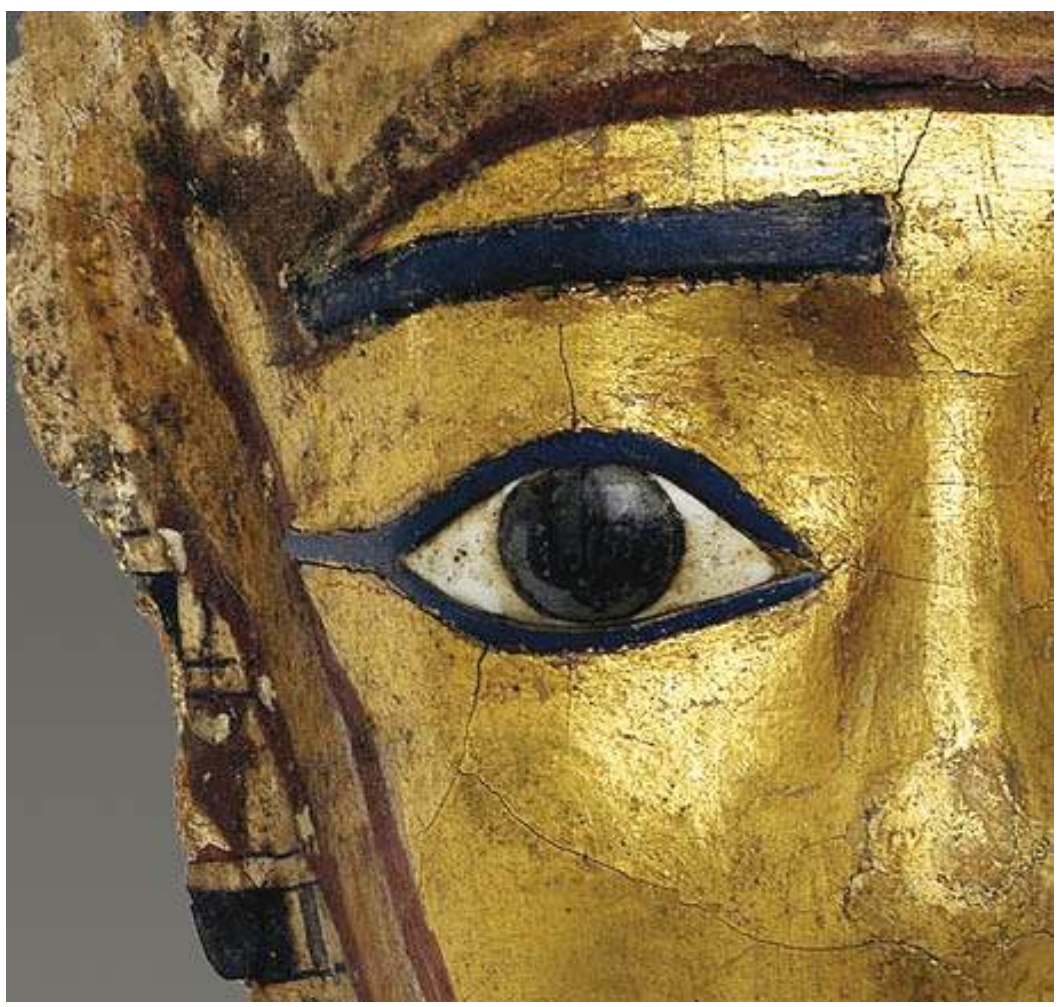

(c) Musée royal de Mariemont/Michel Lechien

Derrière ces mythes et témoignages se cache une réalité, un produit cosmétique que l'on retrouve encore dans des flacons conservés dans les musées, et que l'on peut, grâce aux technologies modernes, analyser et dont les résultats sont disponibles sur Internet. Ce produit, toujours utilisé aujourd'hui, permet de mettre en perspective sa fabrication et ses usages, tant dans l'Antiquité qu'à l'heure actuelle.

\section{Le khôl et la chimie}

18 Durant leurs recherches dans le cadre du concours « Sciences et Beauté », les étudiants ont appliqué les différentes étapes de la démarche scientifique.

\section{Examen de la situation}

19 - observer: les étudiants sont venus au musée et ont reçu du conservateur des collections égyptiennes une médiation ciblée sur les objets qui y sont conservés et qui ont un rapport avec le thème (amulettes, sarcophage, pot à onguents...) ;

20 - analyser un problème : deux des composants du khôl, la galène (sulfure de plomb) et la cérusite (carbonate de plomb) étaient fréquents sur le territoire égyptien, les deux autres, la laurionite (hydroxychlorure de plomb) et la phosgénite (carbonate de plomb) en étaient absents ;

21 - poser une question : comment était fabriqué le khôl en Égypte durant l'Antiquité ? 


\section{Formulation d'une hypothèse} inadéquats, soit les conditions dans lesquelles l'expérience s'est réalisée ne conviennent pas, soit l'équation chimique utilisée est erronée. Un dialogue s'opère alors avec le conservateur de façon à déterminer ce qui a pu conduire à cet échec. Plusieurs éléments peuvent être avancés: tout d'abord, la notion de temps. Les étudiants ont tenté d'obtenir en une heure un résultat qui prenait près de 40 jours aux Égyptiens. Ensuite, l'utilisation de produits purs utilisés en laboratoire et qui ne correspondent pas aux matières premières, non raffinées, disponibles dans l'Antiquité. Ce questionnement aboutit également à une rencontre avec Philippe Walter, ancien directeur des laboratoires des Musées de France, qui a longtemps travaillé sur les résidus de khôl antique conservé dans les musées. Cette rencontre aboutit à un échange fructueux, non seulement du point de vue de la chimie théorique mais également du point de vue pratique et de la manipulation en laboratoire.

Atelier sur le khôl.

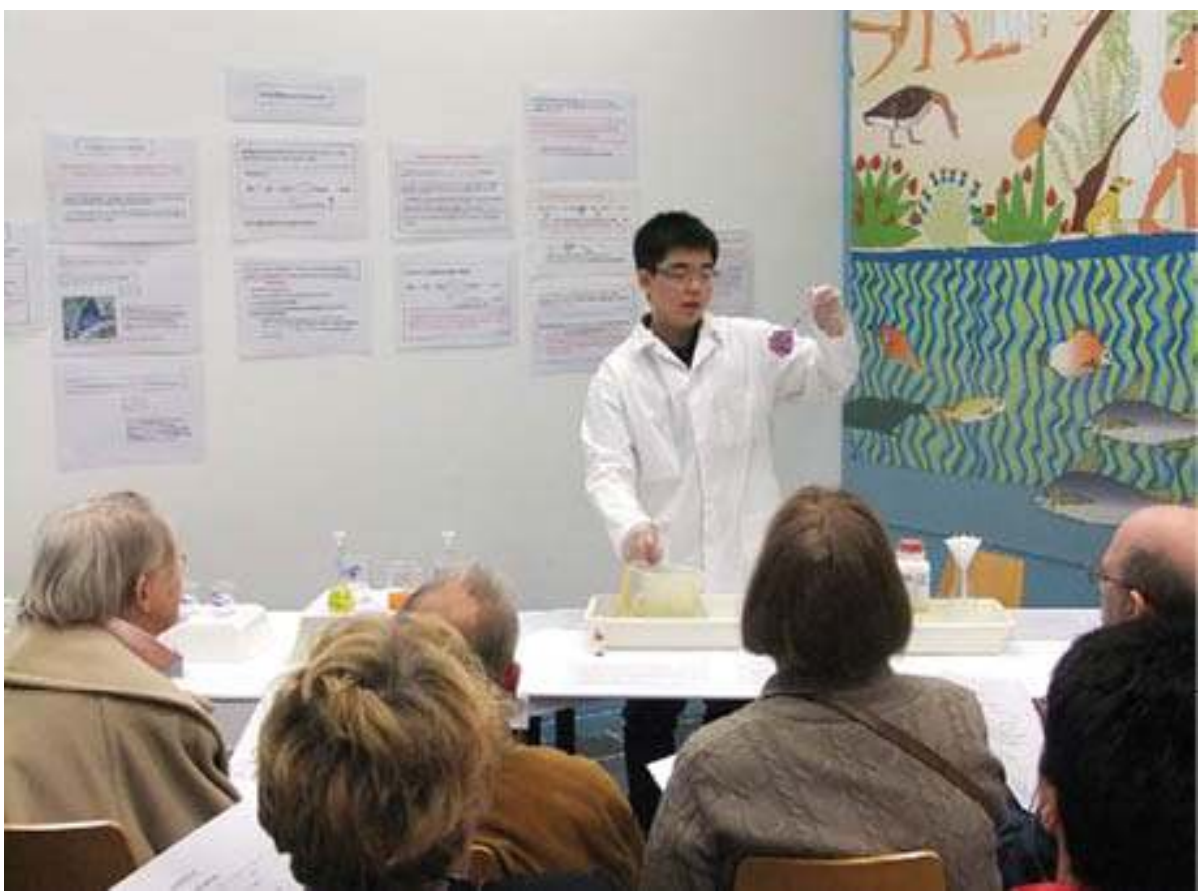

(C) Musée royal de Mariemont 


\section{Réalisation d'une seconde expérience} plusieurs lois et principes chimiques qu'ils avaient étudiés dans leur programme de cours. Ils ont également intégré cette démarche dans une perspective citoyenne et humaine. Ils ont travaillé avec les normes de protection moderne en vigueur dans les laboratoires, là où les anciens Égyptiens manipulaient les éléments de plomb à mains nues.

31 L'aventure ne s'est pas arrêtée là. Ces étudiants, ayant gagné le concours organisé par «Destination Sciences", ont décidé de présenter leur projet devant les Jeunesses scientifiques de Belgique lors de l'EXPOsciences qui s'est déroulée en avril 2014. À l'issue de cette exposition scientifique, ils ont terminé seconds lors du classement final et ont alors représenté la Belgique à un concours européen qui s'est déroulé en Slovaquie à l'automne 2014.

\section{Une visite en tandem}

Suite à cette expérience, nous avons décidé d'organiser une visite en tandem de la collection égyptienne. En compagnie d'un égyptologue, les visiteurs ont parcouru les collections à la rencontre des objets conservés au musée (amulettes, sarcophage, pot à onguents...) de façon à découvrir ce que les sciences historiques connaissent de l'oeil égyptien et du khôl. Ensuite, les étudiants chimistes ont expliqué les procédés et les techniques de "synthétisation chimique " utilisés par les anciens Égyptiens pour réaliser ce produit. En raison de la toxicité des matières premières (à base de plomb), une expérience chimique alternative a été développée. Celle-ci, par le biais de substances colorantes a permis d'expliquer comment les anciens Égyptiens arrivaient à produire les substances qui leur manquaient, notamment en passant d'un milieu acide à un milieu basique.

Deux phases peuvent donc être identifiées au sein du projet : dans un premier temps, le professeur de chimie et l'égyptologue s'associent pour transmettre leurs savoirs respectifs aux élèves, de façon à créer des synergies. Dans un second temps, les 
étudiants, grâce à l'application de la démarche scientifique, s'approprient ces savoirs et s'associent à l'égyptologue pour les communiquer (lors de la visite du musée et lors des concours scientifiques).

La notion de tandem est fondamentale : elle nourrit les visites plus traditionnelles et permet de mettre en dialogue à la fois le médiateur muséal, l'enseignant et les étudiants. Ce type de partenariat demande du temps et des ressources humaines et financières. Néanmoins, nous essayons d'exploiter le résultat obtenu par le biais d'une formule permanente.

\section{Perspectives pour la recherche de nouveaux publics}

Fort du succès de cette expérience relative au khôl, nous avons décidé de proposer une formule similaire aux classes de chimie de l'enseignement secondaire. Celle-ci sera assurée par les médiateurs du service pédagogique qui devront acquérir de nouvelles compétences dans le cadre de leur formation continue. Le partenariat avec l'enseignant prendra la forme de séquences de cours sur la synthétisation chimique des composants du khôl, avant et après la visite au musée. On peut également imaginer que les élèves partagent leurs résultats avec d'autres à l'occasion d'une exposition dans leur école par exemple.

Notre façon de pratiquer la médiation muséale doit donc être repensée, notamment par la rédaction des documents pédagogiques répondant à une nouvelle dynamique. Cette expérience est une illustration de recherche d'autres publics et de nouveaux dispositifs de transmission. Dans le domaine scolaire, cela passe par un partenariat de réciprocitét Ce type de partenariat demande beaucoup d'investissement lors de la création de l'activité. Néanmoins, forte de ce dialogue entre l'enseignant et le médiateur muséal, l'équipe éducative peut mettre au point des visites spécialisées qui présentent à l'enseignant une fiche récapitulative des compétences mises en œuvre tant au musée qu'à l'école et la façon dont l'enseignant peut organiser ses séquences de cours autour de cette thématique. En nous basant sur ce cas précis, en invitant la chimie au musée, nous nous ouvrons à d'autres branches que l'histoire, le latin et le grec... Mais n'est-ce pas là une évolution logique des musées? 
Visite guidée autour de l'utilisation du khôl en Égypte ancienne.

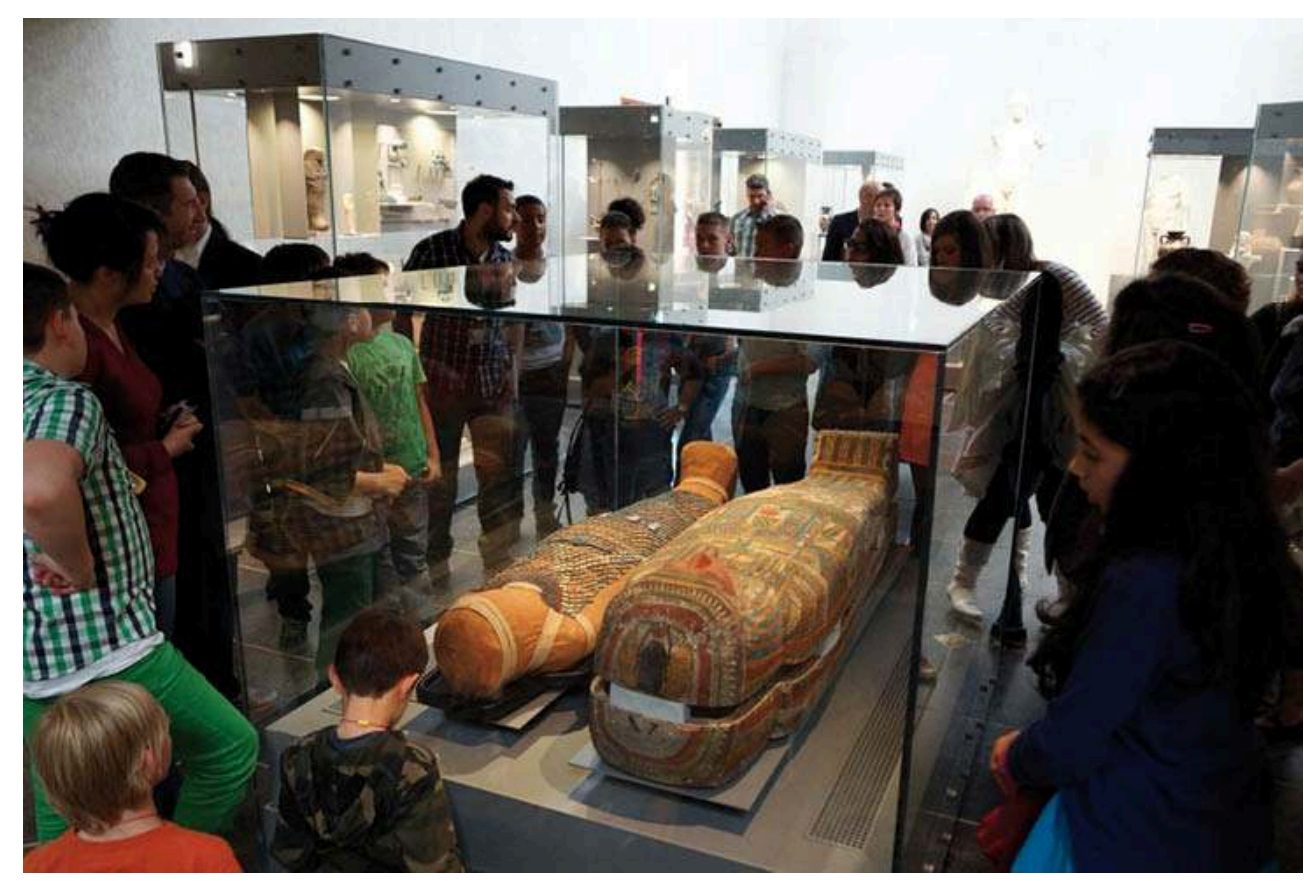

(c) Musée royal de Mariemont

37 Quel public pour quel musée ? Si nous prenons l'exemple d'un musée d'archéologie, on se rend très vite compte que cette discipline ouvre la voie vers les sciences exactes qui lui sont partenaires (notamment l'archéométrie). Nous pouvons alors explorer les collections de façon pluridisciplinaire. Un exemple de cette démarche scientifique et pédagogique sera prochainement mis en œuvre lors de la réouverture du Préhistosite de Ramioul (Flémalle-Liège, Belgique). Dans le cadre du redéploiement de son offre culturelle et touristique, la médiation et la pluridisciplinarité seront au cœur de leur projet. L'équipe pédagogique pourra proposer des expositions-expériences, comme par exemple, «chercher l'homme derrière l'outil»; «le laboratoire d'archéologie expérimentale ( (démonstrations et chantiers de reconstitution); «le Foyer, petits plats grande Histoire ». Tout ceci se retrouve dans une devise de Confucius revendiquée par le Préhistosite de Ramioul : «J'entends, j'oublie ; je vois, je comprends ; je fais, je retiens $»^{5}$. 
Animation avec des scolaires consacrée à l'Égypte ancienne.

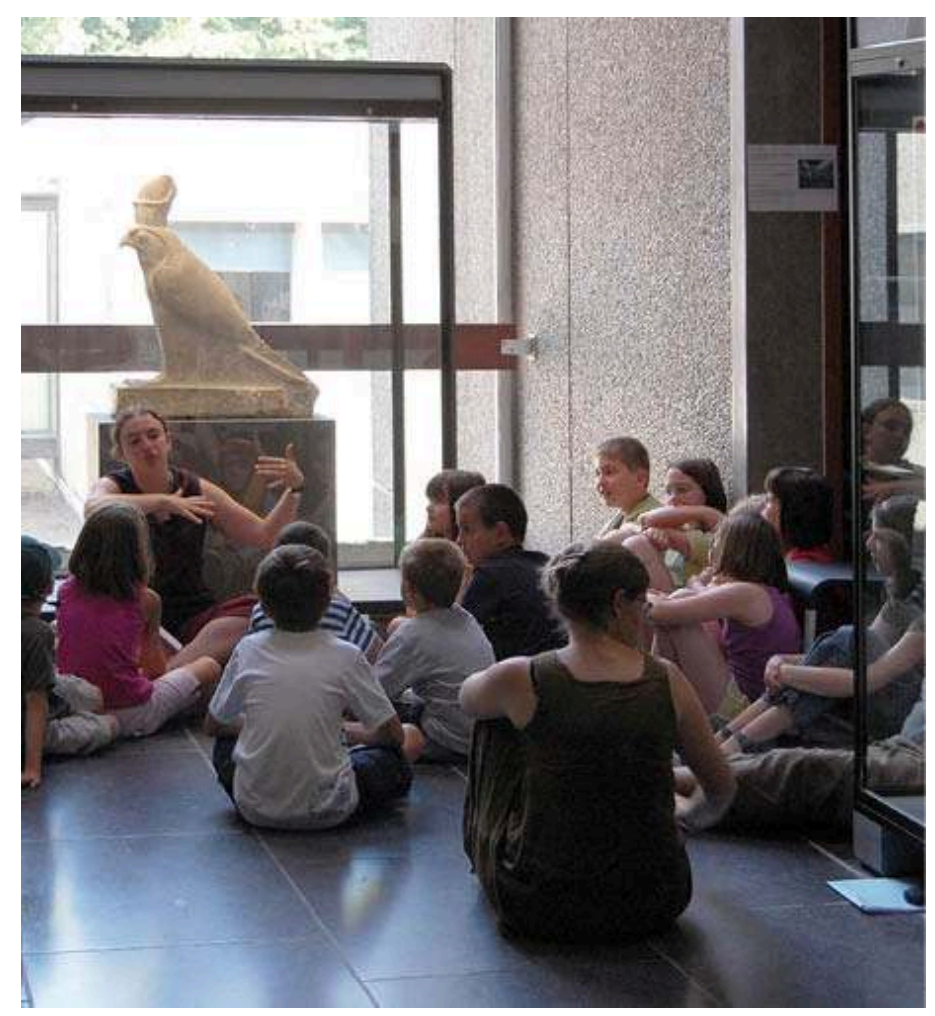

() Musée royal de Mariemont

38 Un projet actuellement en cours au musée royal de Mariemont nous permet d'approfondir la devise. Celui-ci bénéficie du soutien financier de la cellule cultureEnseignement de la Fédération Wallonie-Bruxelles. Il vise à associer la formation historique et scientifique de l'enseignement primaire avec le multimédia. Depuis le réaménagement des collections, le patio du premier étage du musée accueille des vitrines didactiques dont certaines sont dévolues aux techniques de fabrication d'objets en verre, pierre, métal ou encore en terre. Notre souhait est de réaliser un support de médiation adapté permettant aux visiteurs d'appréhender ces différentes techniques au départ des objets présentés. Une collaboration avec une école de La Louvière nous permettra de réaliser une borne tactile. Ce projet se structure en quatre temps :

39 - les élèves du primaire viennent au musée pour découvrir les objets et s'entretenir avec le conservateur du département ainsi qu'avec un médiateur ;

40 - ils vont ensuite à la rencontre d'artisans travaillant ces différents matériaux de façon à appréhender un savoir-faire et à l'expérimenter. Ils vont, par exemple, reproduire les techniques de taille de la pierre ou le montage de céramique ;

41 - de retour en classe, ils doivent trier les différentes informations apprises et mettre en avant celles qui leurs semblent essentielles pour réaliser la borne ;

42 - dans le cadre d'un projet pilote portant sur les écoles numériques, ils réalisent cette borne tactile.

43 Ce type de projet est enthousiasmant. Il nous force à une remise en question qui permet de jeter des ponts entre les disciplines et les catégories de public. Ce sont ainsi les différents enseignants de l'école qui sont mobilisés. On ne peut d'ailleurs que plaider pour l'intégration de la visite du musée au sein des programmes scolaires. Il est 
essentiel que les enseignants s'approprient les collections et puissent, notamment en tandem avec les médiateurs muséaux, explorer les multiples possibilités offertes par le musée.

Ce n'est que par le biais de ces activités, pluridisciplinaires, que nous pourrons pleinement répondre à cette mission prioritaire et essentielle qu'est la médiation du patrimoine auprès du plus grand nombre.

\section{Le musée royal de Mariemont}

(c) Musée royal de Mariemont

Le musée royal de Mariemont doit son existence à Raoul Warocqué (1870-1917). Dernier descendant d'une riche famille d'industriels, il fait partie de ces grands collectionneurs philanthropes de la fin du XIXe et du début du XXe siècle qui prônent une culture accessible au plus grand nombre. Son intérêt se manifeste principalement pour les antiquités extrême-orientales, méditerranéennes, régionales ainsi qu'envers la bibliophilie.

Le décès de Raoul Warocqué en 1917 met temporairement un terme au développement des acquisitions pour Mariemont. Sans héritier, il lègue son domaine, son château et ses collections à l'État belge pour qu'il devienne un musée public. Celui-ci ouvre ses portes en 1920.

Le jour de Noël 1960, un incendie ravage le corps de logis du château. La collection d'œuvres liées à l'histoire régionale et le "Musée de famille » sont irrémédiablement détruits. Certaines pièces bibliophiliques ainsi que des porcelaines de Tournai ont également énormément souffert. Fort heureusement, la part la plus importante des collections se situait dans les deux ailes. Celles-ci, construites en béton armé, ont mieux résisté au feu et sont demeurées pratiquement intactes. Sur les ruines du château sera construit le musée actuel. Ouvert en 1975, il est l'œuvre de Roger Bastin et s'inspire des théories de l'architecte français Le Corbusier.

Devenu établissement scientifique de la Fédération Wallonie-Bruxelles, le musée royal de Mariemont accueille en moyenne plus de 30000 visiteurs par an dont près de 12000 scolaires.

\section{BIBLIOGRAPHIE}

Allard, M. et Lefebvre, B. Le Musée, un lieu éducatif. Montréal, 1997.

Bardinet, T. Les papyrus médicaux de l'Égypte pharaonique. Paris, 1995.

Émond, A.-M. (éd.), L'éducation muséale vue du Canada, des États-Unis et d'Europe : Recherche sur les programmes et les expositions. Montréal, 2006.

Nyst, N., Dupont, C. et Ricker, M.-É. (coord.) Médiation muséale et patrimoniale. Enjeux et perspectives. Bruxelles, 2015. 
Wozny, D. et Simoes, I. (éd.) Parfums et cosmétiques dans l'Égypte ancienne. Catalogue d'exposition, musée d'Archéologie Méditerranéenne, du 6 avril au 23 juin 2002, Paris, 2002.

\section{NOTES}

1. Page Internet du service pédagogique du musée : www.musee-mariemont.be/index.php? id $=12169$

2. Ricker M.-É. et Deceuninck I. Musée : mode d'emploi » enseignant et guide : quelle collaboration ? in Émond, A.-M. (éd.), L'éducation muséale vue du Canada, des États-Unis et d'Europe : recherche sur les programmes et les expositions. Montréal, 2006, p. 202.

3. Pour plus d'information : Fukagawa, S. Investigation into dynamics of ancient Egyptian pharmacology: a statistical analysis of papyrusEbers and cross-cultural medical thinking. Oxford : British Archaeological Reports, BAR International Series 2272, 2011.

4. Partenariat de réciprocité comme défini par Vadeboncœur, G. Le musée et l'école : de la collaboration au partenariat, in Allard, M. et Lefebvre, B. Le Musée, un lieu éducatif. Montréal, 1997, p. 55.

5. Colin, F. et Wéra, M. Le Musée-Médiateur. Les enjeux de la médiation au Préhistosite de Ramioul en chantier (Flémalle-Liège, Belgique), in Nyst, N., Dupont, C. et Ricker, M.-É. (coord.) Médiation muséale et patrimoniale. Enjeux et perspectives. Bruxelles, 2015, pp. 155-164.

\section{RÉSUMÉS}

À partir d'un projet pédagogique mené autour du khôl égyptien par un enseignant en chimie et ses étudiants, le musée royal de Mariemont en Belgique a entrepris une réflexion sur la manière de pratiquer la médiation muséale avec la mise en place d'ateliers multidisciplinaires - mêlant science et histoire - et l'expérimentation de nouveaux dispositifs de transmission, l'ensemble s'inscrivant dans une stratégie globale de développement des publics.

\section{INDEX}

Mots-clés : public, musée

\section{AUTEURS}

\section{ARNAUD QUERTINMONT}

conservateur du département Égypte/Proche-Orient au musée royal de Mariemont arnaud.quertinmont@musee-mariemont.be

\section{MARIE-AUDE LAOUREUX}

responsable du service pédagogique au musée royal de Mariemont marie-aude.laoureux@musee-mariemont.be 